\title{
Analytical Solutions for the Nonlinear Partial Differential Equations Using the Conformable Triple Laplace Transform Decomposition Method
}

\author{
Shailesh A. Bhanotar ${ }^{1}{ }^{1}$ and Mohammed K. A. Kaabar $\mathbb{D i D}^{2,3,4}$ \\ ${ }^{1}$ Department of Mathematics, LJIET, LJ University, Ahmedabad, Gujarat, India \\ ${ }^{2}$ Institute of Mathematical Sciences, Faculty of Science, University of Malaya, Kuala Lumpur 50603, Malaysia \\ ${ }^{3}$ Gofa Camp, Near Gofa Industrial College and German Adebabay, Nifas Silk-Lafto, 26649 Addis Ababa, Ethiopia \\ ${ }^{4}$ Jabalia Camp, United Nations Relief and Works Agency (UNRWA) Palestinian Refugee Camp, \\ Gaza Strip Jabalya, State of Palestine \\ Correspondence should be addressed to Mohammed K. A. Kaabar; mohammed.kaabar@wsu.edu
}

Received 1 April 2021; Accepted 29 July 2021; Published 18 August 2021

Academic Editor: Mostafa Eslami

Copyright ( ${ }^{2} 2021$ Shailesh A. Bhanotar and Mohammed K. A. Kaabar. This is an open access article distributed under the Creative Commons Attribution License, which permits unrestricted use, distribution, and reproduction in any medium, provided the original work is properly cited.

\begin{abstract}
In this paper, a novel analytical method for solving nonlinear partial differential equations is studied. This method is known as triple Laplace transform decomposition method. This method is generalized in the sense of conformable derivative. Important results and theorems concerning this method are discussed. A new algorithm is proposed to solve linear and nonlinear partial differential equations in three dimensions. Moreover, some examples are provided to verify the performance of the proposed algorithm. This method presents a wide applicability to solve nonlinear partial differential equations in the sense of conformable derivative.
\end{abstract}

\section{Introduction}

Fractional calculus has attracted many researchers in the last decades. The impact of this fractional calculus on both pure and applied branches of science and engineering has been increased. Many researchers started to approach with the discrete versions of this fractional of calculus which are summarized into two approaches: nonlocal (classical) and local. Most popular definitions in the area of nonlocal fractional calculus are the Riemann-Liouville, Caputo, and Grunwald-Letnikov definitions. The obtained fractional derivatives lack some basic properties such as chain rule and Leibniz rule for derivatives [1]. However, the semigroup properties of these fractional operators behave well in some cases. In [2], later on, Khalil et al. (2014) presented a new definition of a local fractional derivative, known as conformable derivative, which is well behaved and obeys the Leibniz rule and chain rule for derivatives. While conformable derivative has been criticized in [3,4], we believe that the new definition deserves to be explored further with its analysis and applications because many research studies have been conducted on this definition and its applications to various phenomena in physics and engineering. Therefore, throughout this paper, we will call this definition as conformable derivative. It is defined as follows.

For a function $f:(0, \infty) \longrightarrow R$, the conformable derivative of order $\alpha \in(0,1]$ of $f$ at $x>0$ is defined by

$$
T^{\alpha} f(x)=\lim _{h \longrightarrow 0} \frac{f\left(x+h x^{1-\alpha}\right)-f(x)}{h} .
$$

For this derivative, Atanganana et al. (2018) presented new properties [5] which have been analysed for real valued multivariable functions [6] by Gozutok et al. (2018). In [7], conformable gradient vectors are defined, and a conformable sense Clairaut's theorem has also been proven. In [8-14], the researchers have worked on the linear ordinary and partial differential equations based on the conformable derivatives. Namely, two new results on homogeneous functions involving their conformable partial derivatives are 
introduced, specifically, homogeneity of the conformable partial derivatives of a homogeneous function and the conformable version of Euler's theorem. The conformable Laplace transform was studied and modified by Jarad et al. (2019) [15]. The conformable double Laplace transform was defined and applied in [16]. The conformable Laplace transform is not only useful to solve local conformable fractional dynamical systems but also it can be employed to solve systems within nonlocal conformable fractional derivatives that were defined and used in [17]. Finally, it is also a remarkable fact that there are a large number of studies in the theory and application of fractional differential equations based on this new definition of derivative, which have been developed in a short time. We refer to $[4,18-35]$ that many researchers have been worked on different analogues methods to solve partial fractional differential in conformable sense. Numerical and analytical techniques for solving conformable partial differential equations and conformable initial boundary value problems have been investigated in $[36,37]$, respectively. In addition, some interesting problems have been studied in the sense of conformable derivative such as conformable gradient-based dynamical system for constrained optimization problem [38], conformable heat equation on radial symmetric plate [39], and optimal control problem for conformable heat conduction equation [40]. Several equations have been formulated with the help of conformable formulations to study their solutions such as the $(2+1)$-dimensional Ablowitz-Kaup-Newell-Segur equations [25] where the complex soliton solutions were investigated and the Date-Jimbo-Kashiwara-Miwa equation [26] where new travelling wave solutions were obtained. In addition, the conformable formulations of the coupled nonlinear Schrodinger equations [30] and Fokas-Lenells equation [35] were studied to obtain travelling wave solutions and optical solutions, respectively. The triple Laplace Adomian decomposition method and modified variational iteration Laplace transform method were studied in $[27,28]$, respectively. Similarly, a combined method of both of the Laplace transform and resolvent kernel methods was introduced in [31]. Motivated by all these studies, we come up with the idea to study the nonlinear partial fractional differential equations by defining a function in 3-dimensional space. Therefore, a conformable triple Laplace transform is defined and coupled with Adomian decomposition method to solve systematic nonlinear partial fractional differential equations. The triple Laplace transform has been rarely discussed in the literature which makes this topic as an open research topic. Therefore, exploring new results concerning this interesting topic is always important. The main advantage of this method will give accurate solutions to nonlinear partial fractional derivatives in three-dimensional space.

This paper is divided into the following sections. In Section 2, some basic definitions on conformable partial derivatives are introduced. In Section 3, the main results and theorems on the conformable triple Laplace transform are investigated. In Section 4, a general nonlinear nonhomogeneous partial fractional differential equation is solved using the proposed method. In Section 5, numerical experiment is conducted using the proposed method to validate the obtained results. In Section 6, a conclusion of our research work is provided.

\section{Basic Definitions and Tools}

In this section, we provide some fundamental definitions on conformable partial derivatives.

Definition 1. Given a function $f: R^{+} \times R^{+} \times R^{+} \longrightarrow R$, the conformable partial fractional derivatives (CPFDs) of orders $\alpha, \beta$, and $\gamma$ of the function $f(x, y, t)$ are defined as follows:

$$
\begin{aligned}
& \partial_{x}^{\alpha} f=\frac{\partial^{\alpha}}{\partial x^{\alpha}} f(x, y, t)=\lim _{h \longrightarrow 0} \frac{f\left(x+h x^{1-\alpha}, y, t\right)-f(x, y, t)}{h}, \\
& \partial_{y}^{\beta} f=\frac{\partial^{\beta}}{\partial y^{\beta}} f(x, y, t)=\lim _{k \longrightarrow 0} \frac{f\left(x, y+k y^{1-\beta}, t\right)-f(x, y, t)}{k}, \\
& \partial_{t}^{\gamma} f=\frac{\partial^{\gamma}}{\partial t^{\gamma}} f(x, y, t)=\lim _{\varepsilon \longrightarrow 0} \frac{f\left(x, y, t+\varepsilon t^{1-\gamma}\right)-f(x, y, t)}{\varepsilon},
\end{aligned}
$$

where $0<\alpha, \beta, \gamma \leq 1, x, y, t>0$, and $\partial_{x}^{\alpha}=\left(\partial^{\alpha} / \partial x^{\alpha}\right), \partial_{y}^{\beta}$ $=\left(\partial^{\beta} / \partial y^{\beta}\right)$ and $\partial_{t}^{\gamma}=\left(\partial^{\gamma} / \partial t^{\gamma}\right)$ are called the fractional partial derivatives of orders $\alpha, \beta$, and $\gamma$, respectively.

We prove the basic Theorem 1 and the relation between the CPFDs and partial derivatives as follows.

Theorem 1. Let $\alpha, \beta, \gamma \in(0,1]$ and $f(x, y, t)$ be a differentiable at a point for $x, y, t>0$. Then,

$$
\begin{aligned}
& \text { (i) } \partial_{x}^{\alpha} f=\left(\partial^{\alpha} / \partial x^{\alpha}\right) f(x, y, t)=x^{1-\alpha}(\partial f(x, y, t) / \partial x)= \\
& x^{1-\alpha} \partial_{x} f \\
& \text { (ii) } \partial_{y}^{\beta} f=\left(\partial^{\beta} / \partial y^{\beta}\right) f(x, y, t)=y^{1-\beta}(\partial f(x, y, t) / \partial y)= \\
& y^{1-\beta} \partial_{y} f \\
& \text { (iii) } \partial_{t}^{\gamma} f=\left(\partial^{\gamma} / \partial t^{\gamma}\right) f(x, y, t)=t^{1-\gamma}(\partial f(x, y, t) / \partial t)= \\
& t^{1-\gamma} \partial_{t} f
\end{aligned}
$$

Proof. By the definition of CFPD,

$$
\begin{aligned}
\frac{\partial^{\alpha}}{\partial x^{\alpha}} f(x, y, t)= & \lim _{h \longrightarrow 0} \frac{f\left(x+h x^{1-\alpha}, y, t\right)-f(x, y, t)}{h}, \\
& \text { taking } h x^{1-\alpha}=\varphi \\
= & \lim _{\varphi \longrightarrow 0} \frac{f(x+\varphi, y, t)-f(x, y, t)}{\varphi x^{\alpha-1}} \\
= & x^{1-\alpha} \lim _{\varphi \longrightarrow 0} \frac{f(x+\varphi, y, t)-f(x, y, t)}{\varphi} \\
= & x^{1-\alpha} \frac{\partial f(x, y, t)}{\partial x} .
\end{aligned}
$$

Similarity, we can prove the results (ii) and (iii). 
In the next preposition, we mention the conformable partial fractional derivative of some functions. By using Theorem 1, it can be verified easily.

Proposition 1. Let $\alpha, \beta, \gamma \in(0,1]$ and $a, b \in R, l, m, n \in N$. Then, we have the following:

(1) $\left(\partial^{\alpha} / \partial x^{\alpha}\right)(a u(x, y, t)+b v(x, y, t))=a\left(\partial^{\alpha} / \partial x^{\alpha}\right) u(x$, $y, t)+b\left(\partial^{\alpha} / \partial x^{\alpha}\right) v(x, y, t)$

(2) $\left(\partial^{\alpha+\beta+\gamma} / \partial x^{\alpha} \partial y^{\beta} \partial t^{\gamma}\right)\left(x^{l} y^{m} t^{n}\right)=\operatorname{lm} n x^{l-\alpha} y^{m-\beta} t^{n-\gamma}$

(3) $\left(\partial^{\alpha} / \partial x^{\alpha}\right)\left(\left(x^{\alpha} / \alpha\right)^{l}\left(t^{\gamma} / \gamma\right)^{n}\right)=l\left(x^{\alpha} / \alpha\right)^{l-1}\left(t^{\gamma} / \gamma\right)^{n}, \quad\left(\partial^{\gamma} /\right.$ $\left.\partial t^{\gamma}\right)\left(\left(x^{\alpha} / \alpha\right)^{l}\left(y^{\beta} / \beta\right)^{m}\left(t^{\gamma} / \gamma\right)^{n}\right)=n\left(x^{\alpha} / \alpha\right)^{l}\left(y^{\beta} / \beta\right)^{m}$ $\left(t^{\gamma} / \gamma\right)^{n-1}$

(4) $\left(\partial^{\alpha} / \partial x^{\alpha}\right)\left(\sin \left(x^{\alpha} / \alpha\right) \cos \left(t^{\gamma} / \gamma\right)\right)=\cos \left(x^{\alpha} / \alpha\right) \cos \left(t^{\gamma} / \gamma\right)$

(5) $\left(\partial^{\beta} / \partial y^{\beta}\right)\left(\sin \left(x^{\alpha} / \alpha\right) \cos \left(y^{\beta} / \beta\right) \cos \left(t^{\gamma} / \gamma\right)\right)=-\sin \left(x^{\alpha} /\right.$ $\alpha) \sin \left(y^{\beta} / \beta\right) \cos \left(t^{\gamma} / \gamma\right)$

\section{Some Results and Theorems of the Conformable Triple Laplace Transform}

In this section, we recall some basic definitions on conformable Laplace transform and some results which will be used later on. We refer the reader to some related research studies in $[4,15-29]$. Also, we define conformable triple Laplace transform, which is defined in equation (6).

Definition 2. Let the function $u:(0, \infty) \longrightarrow R$ and $0<\alpha \leq 1$ be the piecewise continuous function. Then, the conformable Laplace transform (CLT) of function $u$ of exponential of order $\alpha$ is defined and denoted by

$$
U_{\alpha}(p)=L_{x}^{\alpha}(u(x))=\int_{0}^{\infty} e^{-p\left(x^{\alpha} / \alpha\right)} u(x) x^{\alpha-1} \mathrm{~d} x, \quad x>0 .
$$

Definition 3. Let $u(x, y)$ be a piecewise continuous function on the domain $\mathrm{D}$ of $R^{+} \times R^{+}$of exponential order $\alpha$ and $\beta$. Then conformable double Laplace Transform (CDLT) of $u(x, y)$ is defined and denoted by

$$
L_{x}^{\alpha} L_{y}^{\beta}(u(x, y))=U_{\alpha, \beta}(p, q)=\int_{0}^{\infty} \int_{0}^{\infty} e^{-p\left(x^{\alpha} / \alpha\right)-q\left(y^{\beta} / \beta\right)} u(x, y) x^{\alpha-1} y^{\beta-1} \mathrm{~d} x \mathrm{~d} y,
$$

where $x, y>0, p, q \in \mathcal{C}, \alpha, \beta \in(0,1]$.

Now, we define the conformable triple Laplace transform, for $\alpha, \beta, \gamma \in(0,1]$, and $p, q, s \in \mathcal{C}$ are the Laplace variables.
Definition 4. Let $u(x, y, t)$ be a real valued piecewise continuous function of $x, y$, and $t$ defined on the domain $\mathrm{D}$ of $R^{+} \times R^{+} \times R^{+}$of exponential order $\alpha, \beta$, and $\gamma$, respectively. Then, the conformable triple Laplace transform (CTLT) of $u(x, y, t)$ is defined as follows:

$$
L_{x}^{\alpha} L_{y}^{\beta} L_{t}^{\gamma}(u(x, y, t))=U_{\alpha, \beta, \gamma}(p, q, s)=\int_{0}^{\infty} \int_{0}^{\infty} \int_{0}^{\infty} e^{-p\left(x^{\alpha} / \alpha\right)-q\left(y^{\beta} / \beta\right)-s\left(t^{\gamma} / \gamma\right)} u(x, y, t) x^{\alpha-1} y^{\beta-1} t^{\gamma-1} \mathrm{~d} x \mathrm{~d} y \mathrm{~d} t,
$$

where $p, q, s \in \mathcal{C}$ are Laplace variables and $\alpha, \beta, \gamma \in(0,1]$.

The conformable inverse triple Laplace transform, denoted by $u(x, y, t)$, is defined by

$$
\begin{aligned}
u(x, y, t) & =L_{p}^{-1} L_{q}^{-1} L_{s}^{-1}\left(U_{\alpha, \beta, \gamma}(p, q, s)\right) \\
& =\frac{1}{2 \pi i} \int_{\alpha-i \infty}^{\alpha+i \infty} e^{p\left(x^{\alpha} / \alpha\right)}\left[\frac{1}{2 \pi i} \int_{\beta-i \infty}^{\beta+i \infty} e^{q\left(y^{\beta} / \beta\right)}\left[\frac{1}{2 \pi i} \int_{\gamma-i \infty}^{\gamma+i \infty} e^{s\left(t^{\gamma} / \gamma\right)} U_{\alpha, \beta, \gamma}(p, q, s) \mathrm{d} s\right] \mathrm{d} q\right] \mathrm{d} p .
\end{aligned}
$$

Definition 5. A unit step or Heaviside unit step function is defined as follows:

$$
H\left(\left(\frac{x^{\alpha}}{\alpha}\right)-a,\left(\frac{y^{\beta}}{\beta}\right)-b,\left(\frac{t^{\gamma}}{\gamma}\right)-c\right)= \begin{cases}1, & x>a, y>b, t>c \\ 0, & x<a, y<b, t<c\end{cases}
$$


Theorem 2. If $L_{x}^{\alpha} L_{y}^{\beta} L_{t}^{\gamma}\left(u\left(x^{\alpha} / \alpha, y^{\beta} / \beta, t^{\gamma} / \gamma\right)\right)=U_{\alpha, \beta, \gamma}(p, q, s)$, $L_{x}^{\alpha} L_{y}^{\beta} L_{t}^{\gamma}\left(v\left(x^{\alpha} / \alpha, y^{\beta} / \beta, t^{\gamma} / \gamma\right)\right)=V_{\alpha, \beta, \gamma}(p, q, s)$, and $A, B$, and $C$ are constants, then

(a) Linearity property:

$$
\begin{aligned}
& L_{x}^{\alpha} L_{y}^{\beta} L_{t}^{\gamma}\left(A u\left(\frac{x^{\alpha}}{\alpha}, \frac{y^{\beta}}{\beta}, \frac{t^{\gamma}}{\gamma}\right)+B v\left(\frac{x^{\alpha}}{\alpha}, \frac{y^{\beta}}{\beta}, \frac{t^{\gamma}}{\gamma}\right)\right) \\
& =A L_{x}^{\alpha} L_{y}^{\beta} L_{t}^{\gamma}\left(u\left(\frac{x^{\alpha}}{\alpha}, \frac{y^{\beta}}{\beta}, \frac{t^{\gamma}}{\gamma}\right)\right)+B L_{x}^{\alpha} L_{y}^{\beta} L_{t}^{\gamma}\left(v\left(\frac{x^{\alpha}}{\alpha}, \frac{y^{\beta}}{\beta}, \frac{t^{\gamma}}{\gamma}\right)\right) \\
& =A U_{\alpha, \beta, \gamma}(p, q, s)+B V_{\alpha, \beta, \gamma}(p, q, s) .
\end{aligned}
$$

(b) $L_{x}^{\alpha} L_{y}^{\beta} L_{t}^{\gamma}(C)=\left(C / p q s^{\prime}\right)$, where $C$ is the constant.

(c) $L_{x}^{\alpha} L_{y}^{\beta} L_{t}^{\gamma}\left(\left(x^{\alpha} / \alpha\right)^{l}\left(y^{\beta} / \beta\right)^{m}\right.$

$\left.\left(t^{\gamma} / \gamma\right)^{n}\right)=\left(\Gamma(l+1) \Gamma(m+1) \Gamma(n+1) / p^{l+1} q^{m+1} s^{n+1}\right)$, where $\Gamma(\cdot)$ is the gamma function. Note that $\Gamma(n+1)=n !$, for $n=0,1,2,3, \ldots$

(d) The first shifting theorem for conformable triple Laplace transform:

If $L_{x}^{\alpha} L_{y}^{\beta} L_{t}^{\gamma}\left(u\left(x^{\alpha} / \alpha, y^{\beta} / \beta, t^{\gamma} / \gamma\right)\right)=U_{\alpha, \beta, \gamma}(p, q, s)$, then

$$
L_{x}^{\alpha} L_{y}^{\beta} L_{t}^{\gamma}\left(e^{a\left(x^{\alpha} / \alpha\right)+b\left(y^{\beta} / \beta\right)+c\left(t^{\gamma} / \gamma\right)} u\left(\frac{x^{\alpha}}{\alpha}, \frac{y^{\beta}}{\beta}, \frac{t^{\gamma}}{\gamma}\right)\right)=U_{\alpha, \beta, \gamma}(p-a, q-b, s-c) .
$$

(e) $L_{x}^{\alpha} L_{y}^{\beta} L_{t}^{\gamma}\left(u\left(x^{\alpha} / \alpha, y^{\beta} / \beta, t^{\gamma} / \gamma\right)\right)=U_{\alpha, \beta, \gamma}(p, q, s)$, then

$$
L_{x}^{\alpha} L_{y}^{\beta} L_{t}^{\gamma}\left(\left(\frac{x^{\alpha}}{\alpha}\right)^{l}\left(\frac{y^{\beta}}{\beta}\right)^{m}\left(\frac{t^{\gamma}}{\gamma}\right)^{n} u\left(\frac{x^{\alpha}}{\alpha}, \frac{y^{\beta}}{\beta}, \frac{t^{\gamma}}{\gamma}\right)\right)=(-1)^{l+m+n} \frac{d^{l+m+n}}{\mathrm{~d} p^{l} \mathrm{~d} q^{m} \mathrm{~d} s^{n}}\left(U_{\alpha, \beta, \gamma}(p, q, s)\right) .
$$

(f)

$$
\begin{aligned}
& L_{x}^{\alpha} L_{y}^{\beta} L_{t}^{\gamma}\left(\sin \left(A \frac{x^{\alpha}}{\alpha}\right) \sin \left(B \frac{y^{\beta}}{\beta}\right) \sin \left(C \frac{t^{\gamma}}{\gamma}\right)\right)=\frac{A B C}{\left(p^{2}+A^{2}\right)\left(q^{2}+B^{2}\right)\left(s^{2}+C^{2}\right)} \\
& L_{x}^{\alpha} L_{y}^{\beta} L_{t}^{\gamma}\left(\cos \left(A \frac{x^{\alpha}}{\alpha}\right) \cos \left(B \frac{y^{\beta}}{\beta}\right) \cos \left(C \frac{t^{\gamma}}{\gamma}\right)\right)=\frac{p q s}{\left(p^{2}+A^{2}\right)\left(q^{2}+B^{2}\right)\left(s^{2}+C^{2}\right)} .
\end{aligned}
$$

Proof. From results (a)-(d) and (f), it can be easily proved by using the definition of conformable triple Laplace transform (CTLT). Here only we see the proof of result (e). So, by the definition of CTLT (equation (6)), we have

$$
\begin{aligned}
L_{x}^{\alpha} L_{y}^{\beta} L_{t}^{\gamma}\left(u\left(\frac{x^{\alpha}}{\alpha}, \frac{y^{\beta}}{\beta}, \frac{t^{\gamma}}{\gamma}\right)\right) & =U_{\alpha, \beta, \gamma}(p, q, s) \\
& =\int_{0}^{\infty} \int_{0}^{\infty} \int_{0}^{\infty}\left(e^{-p\left(x^{\alpha} / \alpha\right)-q\left(y^{\beta} / \beta\right)-s\left(t^{\gamma} / \gamma\right)} u\left(\frac{x^{\alpha}}{\alpha}, \frac{y^{\beta}}{\beta}, \frac{t^{\gamma}}{\gamma}\right) \times x^{\alpha-1} y^{\beta-1} t^{\gamma-1}\right) \mathrm{d} x \mathrm{~d} y \mathrm{~d} t .
\end{aligned}
$$

By differentiating with respect to $p, l$-times, we get 


$$
\begin{aligned}
\frac{\mathrm{d}^{l}}{\mathrm{~d} p^{l}} U_{\alpha, \beta, \gamma}(p, q, s) & =\frac{\mathrm{d}^{l}}{\mathrm{~d} p^{l}}\left\{\int_{0}^{\infty} \int_{0}^{\infty} \int_{0}^{\infty}\left(e^{-p\left(x^{\alpha} / \alpha\right)-q\left(y^{\beta} / \beta\right)-s\left(t^{\gamma} / \gamma\right)} u\left(\frac{x^{\alpha}}{\alpha}, \frac{y^{\beta}}{\beta}, \frac{t^{\gamma}}{\gamma}\right) \times x^{\alpha-1} y^{\beta-1} t^{\gamma-1}\right) \mathrm{d} x \mathrm{~d} y \mathrm{~d} t\right\} \\
& =\int_{0}^{\infty} \int_{0}^{\infty} \int_{0}^{\infty} \frac{\partial^{l}}{d \partial^{l}}\left[e^{-p\left(x^{\alpha} / \alpha\right)-q\left(y^{\beta} / \beta\right)-s\left(t^{\gamma} / \gamma\right)} \times u\left(\frac{x^{\alpha}}{\alpha}, \frac{y^{\beta}}{\beta}, \frac{t^{\gamma}}{\gamma}\right) x^{\alpha-1} y^{\beta-1} t^{\gamma-1}\right] \mathrm{d} x \mathrm{~d} y \mathrm{~d} t .
\end{aligned}
$$

It reduces to

$$
\frac{\mathrm{d}^{l}}{\mathrm{~d} p^{l}} U_{\alpha, \beta, \gamma}(p, q, s)=\int_{0}^{\infty} \int_{0}^{\infty} \int_{0}^{\infty}\left(-\left(\frac{x^{\alpha}}{\alpha}\right)\right)^{l}\left[e^{-p\left(x^{\alpha} / \alpha\right)-q\left(y^{\beta} / \beta\right)-s\left(t^{\gamma} / \gamma\right)} \times u\left(\frac{x^{\alpha}}{\alpha}, \frac{y^{\beta}}{\beta}, \frac{t^{\gamma}}{\gamma}\right) x^{\alpha-1} y^{\beta-1} t^{\gamma-1}\right] \mathrm{d} x \mathrm{~d} y \mathrm{~d} t
$$

Now, we again differentiate with respect to $q$ and $s, m$ and $n$-times, respectively, and we obtain the simplification as follows:

$$
\begin{aligned}
\frac{\mathrm{d}^{l+m+n}}{\mathrm{~d} p^{l} \mathrm{~d} q^{m} \mathrm{~d} s^{n}}\left(U_{\alpha, \beta, \gamma}(p, q, s)\right)= & \int_{0}^{\infty} \int_{0}^{\infty} \int_{0}^{\infty}\left(\left(-\left(\frac{x^{\alpha}}{\alpha}\right)\right)^{l}\left(-\left(\frac{y^{\beta}}{\beta}\right)\right)^{m}\left(-\left(\frac{t^{\gamma}}{\gamma}\right)\right)^{n}\right. \\
& \left.\times e^{-p\left(x^{\alpha} / \alpha\right)-q\left(y^{\beta} / \beta\right)-s\left(t^{\gamma} / \gamma\right)} u\left(\frac{x^{\alpha}}{\alpha}, \frac{y^{\beta}}{\beta}, \frac{t^{\gamma}}{\gamma}\right) \times x^{\alpha-1} y^{\beta-1} t^{\gamma-1}\right) \mathrm{d} x \mathrm{~d} y \mathrm{~d} t \\
= & (-1)^{l+m+n} \int_{0}^{\infty} \int_{0}^{\infty} \int_{0}^{\infty}\left(e^{-p\left(x^{\alpha} / \alpha\right)-q\left(y^{\beta} / \beta\right)-s\left(t^{\gamma} / \gamma\right)}\right. \\
& \left.\times\left(\frac{x^{\alpha}}{\alpha}\right)^{l}\left(\frac{y^{\beta}}{\beta}\right)^{m}\left(\frac{t^{\gamma}}{\gamma}\right)^{n} \times u\left(\frac{x^{\alpha}}{\alpha}, \frac{y^{\beta}}{\beta}, \frac{t^{\gamma}}{\gamma}\right) x^{\alpha-1} y^{\beta-1} t^{\gamma-1}\right) \mathrm{d} x \mathrm{~d} y \mathrm{~d} t,
\end{aligned}
$$

which implies

$$
\frac{\mathrm{d}^{l+m+n}}{\mathrm{~d} p^{l} \mathrm{~d} q^{m} \mathrm{~d} s^{n}}\left(U_{\alpha, \beta, \gamma}(p, q, s)\right)=(-1)^{l+m+n} L_{x}^{\alpha} L_{y}^{\beta} L_{t}^{\gamma}\left(\left(\frac{x^{\alpha}}{\alpha}\right)^{l}\left(\frac{y^{\beta}}{\beta}\right)^{m}\left(\frac{t^{\gamma}}{\gamma}\right)^{n} u\left(\frac{x^{\alpha}}{\alpha}, \frac{y^{\beta}}{\beta}, \frac{t^{\gamma}}{\gamma}\right)\right)
$$

Now, we multiply $(-1)^{l+m+n}$, on both sides, and we get the required result:

$$
L_{x}^{\alpha} L_{y}^{\beta} L_{t}^{\gamma}\left(\left(\frac{x^{\alpha}}{\alpha}\right)^{l}\left(\frac{y^{\beta}}{\beta}\right)^{m}\left(\frac{t^{\gamma}}{\gamma}\right)^{n} u\left(\frac{x^{\alpha}}{\alpha}, \frac{y^{\beta}}{\beta}, \frac{t^{\gamma}}{\gamma}\right)\right)=(-1)^{l+m+n} \frac{\mathrm{d}^{l+m+n}}{\mathrm{~d} p^{l} \mathrm{~d} q^{m} \mathrm{~d} s^{n}}\left(U_{\alpha, \beta, \gamma}(p, q, s)\right) .
$$

Theorem 3. If $L_{x}^{\alpha} L_{y}^{\beta} L_{t}^{\gamma}\left(u\left(x^{\alpha} / \alpha, y^{\beta} / \beta, t^{\gamma} / \gamma\right)\right)=U_{\alpha, \beta, \gamma}(p, q, s)$, then 


$$
\begin{aligned}
& L_{x}^{\alpha} L_{y}^{\beta} L_{t}^{\gamma}\left(u\left(\frac{x^{\alpha}}{\alpha}-\frac{\zeta^{\alpha}}{\alpha}, \frac{y^{\beta}}{\beta}-\frac{\eta^{\beta}}{\beta}, \frac{t^{\gamma}}{\gamma}-\frac{\theta^{\gamma}}{\gamma}\right) \times H\left(\frac{x^{\alpha}}{\alpha}-\frac{\zeta^{\alpha}}{\alpha}, \frac{y^{\beta}}{\beta}-\frac{\eta^{\beta}}{\beta}, \frac{t^{\gamma}}{\gamma}-\frac{\theta^{\gamma}}{\gamma}\right)\right) \\
& =e^{-p\left(\zeta^{\alpha} / \alpha\right)-q\left(\eta^{\beta} / \beta\right)-s\left(\theta^{\gamma} / \gamma\right)} U_{\alpha, \beta, \gamma}(p, q, s),
\end{aligned}
$$

where $H(x, y, t)$ is a Heaviside unit step function as defined Proof. By applying the definition of CTLT, we have in equation (8).

$$
\begin{aligned}
\text { LHS }= & L_{x}^{\alpha} L_{y}^{\beta} L_{t}^{\gamma}\left(u\left(\frac{x^{\alpha}}{\alpha}-\frac{\zeta^{\alpha}}{\alpha}, \frac{y^{\beta}}{\beta}-\frac{\eta^{\beta}}{\beta}, \frac{t^{\gamma}}{\gamma}-\frac{\theta^{\gamma}}{\gamma}\right) H\left(\frac{x^{\alpha}}{\alpha}-\frac{\zeta^{\alpha}}{\alpha}, \frac{y^{\beta}}{\beta}-\frac{\eta^{\beta}}{\beta}, \frac{t^{\gamma}}{\gamma}-\frac{\theta^{\gamma}}{\gamma}\right)\right) \\
= & \int_{0}^{\infty} \int_{0}^{\infty} \int_{0}^{\infty}\left(e^{-p\left(x^{\alpha} / \alpha\right)-q\left(y^{\beta} / \beta\right)-s\left(t^{\gamma} / \gamma\right)} u\left(\frac{x^{\alpha}}{\alpha}-\frac{\zeta^{\alpha}}{\alpha}, \frac{y^{\beta}}{\beta}-\frac{\eta^{\beta}}{\beta}, \frac{t^{\gamma}}{\gamma}-\frac{\theta^{\gamma}}{\gamma}\right)\right. \\
& \left.\times H\left(\frac{x^{\alpha}}{\alpha}-\frac{\zeta^{\alpha}}{\alpha}, \frac{y^{\beta}}{\beta}-\frac{\eta^{\beta}}{\beta}, \frac{t^{\gamma}}{\gamma}-\frac{\theta^{\gamma}}{\gamma}\right) x^{\alpha-1} y^{1-\beta} t^{1-\gamma}\right) \mathrm{d} x \mathrm{~d} y \mathrm{~d} t .
\end{aligned}
$$

Now, using the definition of Heaviside unit step function $H(x, y, t)$, we have

$$
=\int_{\left(\zeta^{\alpha} / \alpha\right)}^{\infty} \int_{\left(\eta^{\beta} / \beta\right)}^{\infty} \int_{\left(\theta^{\gamma} / \gamma\right)}^{\infty}\left(e^{-p\left(x^{\alpha} / \alpha\right)-q\left(y^{\beta} / \beta\right)-s\left(t^{\gamma} / \gamma\right)} \times u\left(\frac{x^{\alpha}}{\alpha}-\frac{\zeta^{\alpha}}{\alpha}, \frac{y^{\beta}}{\beta}-\frac{\eta^{\beta}}{\beta}, \frac{t^{\gamma}}{\gamma}-\frac{\theta^{\gamma}}{\gamma}\right) \times x^{\alpha-1} y^{\beta-1} t^{\gamma-1}\right) \mathrm{d} x \mathrm{~d} y \mathrm{~d} t
$$

By putting $\left(x^{\alpha} / \alpha\right)-\left(\zeta^{\alpha} / \alpha\right)=\left(\mathscr{A}^{\alpha} / \alpha\right), \quad\left(y^{\beta} / \beta\right)-$ $\left(\eta^{\beta} / \beta\right)=\left(\mathscr{T}^{\beta} / \beta\right),\left(t^{\gamma} / \gamma\right)-\left(\theta^{\gamma} / \gamma\right)=\left(\mathcal{U}^{\gamma} / \gamma\right)$, we have

$$
\begin{aligned}
L_{x}^{\alpha} L_{y}^{\beta} L_{t}^{\gamma}\left(u\left(\frac{x^{\alpha}}{\alpha}-\frac{\zeta^{\alpha}}{\alpha}, \frac{y^{\beta}}{\beta}-\frac{\eta^{\beta}}{\beta}, \frac{t^{\gamma}}{\gamma}-\frac{\theta^{\gamma}}{\gamma}\right) H\left(\frac{x^{\alpha}}{\alpha}-\frac{\zeta^{\alpha}}{\alpha}, \frac{y^{\beta}}{\beta}-\frac{\eta^{\beta}}{\beta}, \frac{t^{\gamma}}{\gamma}-\frac{\theta^{\gamma}}{\gamma}\right)\right) \\
=e^{-p\left(x^{\alpha} / \alpha\right)-q\left(y^{\beta} / \beta\right)-s\left(t^{\gamma} / \gamma\right)} \int_{0}^{\infty} \int_{0}^{\infty} \int_{0}^{\infty}\left(e^{-p\left(x^{\alpha} / \alpha\right)-q\left(y^{\beta} / \beta\right)-s\left(t^{\gamma} / \gamma\right)}\right. \\
\left.\quad \times u\left(\frac{\mathscr{A}^{\alpha}}{\alpha}, \frac{\mathscr{T}^{\beta}}{\beta}, \frac{\mathcal{U}^{\gamma}}{\gamma}\right) \times \mathscr{A}^{\alpha-1} \mathscr{T}^{\beta-1} \mathcal{U}^{\gamma-1}\right) \mathrm{d} \mathscr{A} \mathrm{d} \mathscr{T} \mathrm{d} \mathscr{U} \\
=e^{-p\left(\zeta^{\alpha} / \alpha\right)-q\left(\eta^{\beta} / \beta\right)-s\left(\theta^{\gamma} / \gamma\right)} U_{\alpha, \beta, \gamma}(p, q, s) .
\end{aligned}
$$

Theorem 4. The conformable triple Laplace transform of the function $\quad\left(x^{\alpha} / \alpha\right)^{l} u(x, y, t), \quad\left(y^{\beta} / \beta\right)^{m} u(x, y, t),\left(t^{\gamma} / \gamma\right)^{n}$ $u(x, y, t)$ and $\left(\partial^{\beta+\gamma} / \partial y^{\beta} \partial t^{\gamma}\right)(u(x, y, t))$ is given by

(a) $L_{x}^{\alpha} L_{y}^{\beta} L_{t}^{\gamma}\left(\left(x^{\alpha} / \alpha\right)^{l} u\left(\left(x^{\alpha} / \alpha\right),\left(y^{\beta} / \beta\right), \quad\left(t^{\gamma} / \gamma\right)\right)\right)=(-1)^{l}$ $\left(d^{l} / d p^{l}\right)\left(L_{x}^{\alpha} L_{y}^{\beta} L_{t}^{\gamma}\left(u\left(\left(x^{\alpha} / \alpha\right),\left(y^{\beta} / \beta\right), \quad\left(t^{\gamma} / \gamma\right)\right)\right)\right)=$ $(-1)^{l}\left(d^{l} / d p^{l}\right)\left(U_{\alpha, \beta, \gamma}(p, q, s)\right)$

(b) $L_{x}^{\alpha} L_{y}^{\beta} L_{t}^{\gamma}\left(\left(y^{\beta} / \beta\right)^{m} u\left(\left(x^{\alpha} / \alpha\right), \quad\left(y^{\beta} / \beta\right),\left(t^{\gamma} / \gamma\right)\right)\right)=(-1)^{m}$ $\left(d^{m} / d q^{m}\right)\left(L_{x}^{\alpha} L_{y}^{\beta} L_{t}^{\gamma} \quad\left(u\left(x^{\alpha} / \alpha\right),\left(y^{\beta} / \beta\right),\left(t^{\gamma} / \gamma\right)\right)\right)=$ $(-1)^{m}\left(d^{m} / d q^{m}\right)\left(U_{\alpha, \beta, \gamma}(p, q, s)\right)$ (c) $L_{x}^{\alpha} L_{y}^{\beta} L_{t}^{\gamma}\left(\left(t^{\gamma} / \gamma\right)^{n} u\left(\left(x^{\alpha} / \alpha\right),\left(y^{\beta} / \beta\right), \quad\left(t^{\gamma} / \gamma\right)\right)\right)=(-1)^{n}$ $\left(d^{n} / d s^{n}\right)\left(L_{x}^{\alpha} L_{y}^{\beta} L_{t}^{\gamma}\left(u\left(\left(x^{\alpha} / \alpha\right), \quad\left(y^{\beta} / \beta\right),\left(t^{\gamma} / \gamma\right)\right)\right)\right)=$ $(-1)^{n}\left(d^{n} / d s^{n}\right)\left(U_{\alpha, \beta, \gamma}(p, q, s)\right)$

(d) $L_{x}^{\alpha} L_{y}^{\beta} L_{t}^{\gamma}\left(\left(x^{\alpha} / \alpha\right)^{l}\left(\partial^{\beta+\gamma} / \partial y^{\beta} \partial t^{\gamma}\right)\left(u\left(\left(x^{\alpha} / \alpha\right), \quad\left(y^{\beta} / \beta\right)\right.\right.\right.$, $\left.\left.\left.\left(t^{\gamma} / \gamma\right)\right)\right)\right)=(-1)^{l}\left(d^{l} / d p^{l}\right)\left(L_{x}^{\alpha} L_{y}^{\beta} L_{t}^{\gamma} \quad\left(\left(\partial^{\beta+\gamma} / \partial y^{\beta} \partial t^{\gamma}\right)\right.\right.$ $\left.\left.\left(u\left(\left(x^{\alpha} / \alpha\right),\left(y^{\beta} / \beta\right),\left(t^{\gamma} / \gamma\right)\right)\right)\right)\right)$

Proof. By applying the definition of CTLT and Theorem 2 (e), till equation (15) can be the required result (a). 
Remaining results (b)-(d) can be obtained via the same process.

Theorem 5. For $\alpha, \beta, \gamma \in(0,1]$. Let $u(x, y, t)=$ $u\left(x^{\alpha} / \alpha, y^{\beta} / \beta, t^{\gamma} / \gamma\right)$ be the real valued piecewise continuous function $x, y$, and $t$ of the domain $D$ on $(0, \infty)$ $\times(0, \infty) \times(0, \infty)$. The CTLT (conformable triple Laplace transform) of the conformable partial fractional derivatives of order $\alpha, \beta$, and $\gamma$ is given by

(a) $L_{x}^{\alpha} L_{y}^{\beta} L_{t}^{\gamma}\left(\left(\partial^{\alpha} / \partial x^{\alpha}\right)\left(u\left(x^{\alpha} / \alpha, y^{\beta} / \beta, t^{\gamma} / \gamma\right)\right)\right)=p U(p, q$, s) $-U(0, q, s)$

(b) $L_{x}^{\alpha} L_{y}^{\beta} L_{t}^{\gamma}\left(\left(\partial^{\beta} / \partial y^{\beta}\right)\left(u\left(x^{\alpha} / \alpha, y^{\beta} / \beta, t^{\gamma} / \gamma\right)\right)\right)=q U(p, q$, $s)-U(p, 0, s)$

(c) $L_{x}^{\alpha} L_{y}^{\beta} L_{t}^{\gamma}\left(\left(\partial^{\gamma} / \partial t^{\gamma}\right)\left(u\left(x^{\alpha} / \alpha, y^{\beta} / \beta, t^{\gamma} / \gamma\right)\right)\right)=s U(p, q$, $s)-U(p, q, 0)$ (d) $L_{x}^{\alpha} L_{y}^{\beta} L_{t}^{\gamma}\left(\left(\partial^{2 \alpha} / \partial x^{2 \alpha}\right)\left(u\left(x^{\alpha} / \alpha, y^{\beta} / \beta, t^{\gamma} / \gamma\right)\right)\right)=p^{2} U(p$, $q, s)-p U(0, q, s)-U_{x}(0, q, s)$

(e) $L_{x}^{\alpha} L_{y}^{\beta} L_{t}^{\gamma}\left(\left(\partial^{2 \beta} / \partial y^{2 \beta}\right)\left(u\left(x^{\alpha} / \alpha, y^{\beta} / \beta, t^{\gamma} / \gamma\right)\right)\right)=q^{2} U(p$, $q, s)-q U(p, 0, s)-U_{y}(p, 0, s)$

(f) $L_{x}^{\alpha} L_{y}^{\beta} L_{t}^{\gamma}\left(\left(\partial^{2 \gamma} / \partial t^{2 \gamma}\right)\left(u\left(x^{\alpha} / \alpha, y^{\beta} / \beta, t^{\gamma} / \gamma\right)\right)\right)=s^{2} U(p$, $q, s)-s U(p, q, 0)-U_{t}(p, q, 0)$

(g) $L_{x}^{\alpha} L_{y}^{\beta} L_{t}^{\gamma}\left(\left(\partial^{3 \alpha} / \partial x^{3 \alpha}\right)\left(u\left(x^{\alpha} / \alpha, y^{\beta} / \beta, t^{\gamma} / \gamma\right)\right)\right)=\left[p^{3} U(p\right.$, $\left.q, s)-p^{2} U(0, q, s)-p U_{x}(0, q, s)-U_{x x}(0, q, s)\right]$

Proof. Here, we go for proof of result (a), and the remaining results (b)-(g) can be proved. To obtain conformable triple Laplace transform of the fractional partial derivatives, we use integration by parts and Theorem 1 .

By applying the definition of CTLT, we have

$$
\begin{aligned}
L_{x}^{\alpha} L_{y}^{\beta} L_{t}^{\gamma}\left(\frac{\partial^{\alpha}}{\partial x^{\alpha}}\left(u\left(\frac{x^{\alpha}}{\alpha}, \frac{y^{\beta}}{\beta}, \frac{t^{\gamma}}{\gamma}\right)\right)\right) & =\int_{0}^{\infty} \int_{0}^{\infty} \int_{0}^{\infty}\left(e^{-p\left(\zeta^{\alpha} / \alpha\right)-q\left(\eta^{\beta} / \beta\right)-s\left(\theta^{\gamma} / \gamma\right)} \frac{\partial^{\alpha}(u)}{\partial x^{\alpha}} \times x^{\alpha-1} y^{\beta-1} t^{\gamma-1}\right) \mathrm{d} x \mathrm{~d} y \mathrm{~d} t \\
& =\int_{0}^{\infty} \int_{0}^{\infty}\left(e^{-q\left(y^{\beta} / \beta\right)-s\left(t^{\gamma} / \gamma\right)}\left(\int_{0}^{\infty} e^{-p\left(x^{\alpha} / \alpha\right)} \frac{\partial^{\alpha}(u)}{\partial x^{\alpha}} x^{\alpha-1} \mathrm{~d} x\right) \times y^{\beta-1} t^{\gamma-1}\right) \mathrm{d} y \mathrm{~d} t .
\end{aligned}
$$

Since we have Theorem 1, $\left(\partial^{\alpha}(u) / \partial x^{\alpha}\right)=x^{1-\alpha}(\partial u / \partial x)$. We use this result in equation (23).

Therefore, equation (23) becomes

$$
\begin{aligned}
& L_{x}^{\alpha} L_{y}^{\beta} L_{t}^{\gamma}\left(\frac{\partial^{\alpha}}{\partial x^{\alpha}}\left(u\left(\frac{x^{\alpha}}{\alpha}, \frac{y^{\beta}}{\beta}, \frac{t^{\gamma}}{\gamma}\right)\right)\right) \\
& =\int_{0}^{\infty} \int_{0}^{\infty} e^{-q\left(y^{\beta} / \beta\right)-s\left(t^{\gamma} / \gamma\right)}\left(\int_{0}^{\infty} e^{-p\left(x^{\alpha} / \alpha\right)} \frac{\partial u}{\partial x} \mathrm{~d} x\right) y^{\beta-1} t^{\gamma-1} \mathrm{~d} y \mathrm{~d} t .
\end{aligned}
$$

The integral inside the bracket is given by

$$
\int_{0}^{\infty} e^{-p\left(x^{\alpha} / \alpha\right)} \frac{\partial u}{\partial x} \mathrm{~d} x=p U(p, y, t)-U(0, y, t)
$$

By substituting equation (25) in equation (24), and simplifying, we get the required result (a), that is

$$
L_{x}^{\alpha} L_{y}^{\beta} L_{t}^{\gamma}\left(\frac{\partial^{\alpha}}{\partial x^{\alpha}}\left(u\left(\frac{x^{\alpha}}{\alpha}, \frac{y^{\beta}}{\beta}, \frac{t^{\gamma}}{\gamma}\right)\right)\right)=p U(p, q, s)-U(0, q, s) .
$$

In general, the above results in Theorem 5 can be extended as follows:

(a)

$$
\begin{aligned}
L_{x}^{\alpha} L_{y}^{\beta} L_{t}^{\gamma}\left(\frac{\partial^{\alpha+\beta+\gamma}}{\partial x^{\alpha} \partial y^{\beta} \partial t^{\gamma}}\left(u\left(\frac{x^{\alpha}}{\alpha}, \frac{y^{\beta}}{\beta}, \frac{t^{\gamma}}{\gamma}\right)\right)\right)= & (p q s U(p, q, s)-p U(p, 0,0)-q U(0, q, 0)-s U(0,0, s)-p q U(p, q, 0) \\
& -p s U(p, 0, s)-q s U(0, q, s)-U(0,0,0)) .
\end{aligned}
$$

(b) If $L_{x}^{\alpha} L_{y}^{\beta} L_{t}^{\gamma}\left(u\left(x^{\alpha} / \alpha, y^{\beta} / \beta, t^{\gamma} / \gamma\right)\right)=U_{\alpha, \beta, \gamma}(p, q, s)$, then 


$$
L_{x}^{\alpha} L_{y}^{\beta} L_{t}^{\gamma}\left(\frac{\partial^{m}}{\partial t^{m}}\left(u\left(\frac{x^{\alpha}}{\alpha}, \frac{y^{\beta}}{\beta}, \frac{t^{\gamma}}{\gamma}\right)\right)\right)=s^{m} U(p, q, s)-\sum_{k=0}^{m-1} s^{m-1-k} U_{t}^{(k)}(p, q, 0) .
$$

\section{Solving Nonlinear Partial Fractional \\ Differential Equation Using the Conformable \\ Triple Laplace Transform \\ Decomposition Method}

We consider a general nonlinear nonhomogeneous partial fractional differential equation:

$$
\frac{\partial^{\gamma m}}{\partial t^{\gamma m}}\left(u\left(\frac{x^{\alpha}}{\alpha}, \frac{y^{\beta}}{\beta}, \frac{t^{\gamma}}{\gamma}\right)\right)+R u\left(\frac{x^{\alpha}}{\alpha}, \frac{y^{\beta}}{\beta}, \frac{t^{\gamma}}{\gamma}\right)+N u\left(\frac{x^{\alpha}}{\alpha}, \frac{y^{\beta}}{\beta}, \frac{t^{\gamma}}{\gamma}\right)=g\left(\frac{x^{\alpha}}{\alpha}, \frac{y^{\beta}}{\beta}, \frac{t^{\gamma}}{\gamma}\right),
$$

where $m=1,2,3, \ldots$ and $\gamma \in(0,1]$ with the initial conditions

$$
\frac{\partial^{\gamma m-1}}{\partial t^{\gamma m-1}}\left(u\left(\frac{x^{\alpha}}{\alpha}, \frac{y^{\beta}}{\beta}, 0\right)\right)=f_{\gamma m-1}\left(\frac{x^{\alpha}}{\alpha}, \frac{y^{\beta}}{\beta}, 0\right)
$$

where $R$ is the linear differential operator and $N$ addresses the nonlinear partial fractional operator, and $g=g\left(x^{\alpha} / \alpha, y^{\beta} / \beta, t^{\gamma} / \gamma\right)$ is the source term. In order to solve equation (29), we follow the following steps:
Step 1: applying the conformable triple Laplace transform to equation (29) on both sides, we have

$$
\begin{aligned}
& L_{x}^{\alpha} L_{y}^{\beta} L_{t}^{\gamma} \frac{\partial^{\gamma m}}{\partial t^{\gamma m}}\left(u\left(\frac{x^{\alpha}}{\alpha}, \frac{y^{\beta}}{\beta}, \frac{t^{\gamma}}{\gamma}\right)\right)+L_{x}^{\alpha} L_{y}^{\beta} L_{t}^{\gamma}\left(R u\left(\frac{x^{\alpha}}{\alpha}, \frac{y^{\beta}}{\beta}, \frac{t^{\gamma}}{\gamma}\right)\right) \\
& +L_{x}^{\alpha} L_{y}^{\beta} L_{t}^{\gamma}\left(N u\left(\frac{x^{\alpha}}{\alpha}, \frac{y^{\beta}}{\beta}, \frac{t^{\gamma}}{\gamma}\right)\right)=L_{x}^{\alpha} L_{y}^{\beta} L_{t}^{\gamma}\left(g\left(\frac{x^{\alpha}}{\alpha}, \frac{y^{\beta}}{\beta}, \frac{t^{\gamma}}{\gamma}\right)\right) .
\end{aligned}
$$

Using Theorem 5 and equation (30), in equation (31),

$$
\begin{aligned}
& s^{m} U(p, r, s)-\sum_{k=0}^{m-1} s^{m-1-k} U_{t}^{(k)}(p, q, 0)+L_{x}^{\alpha} L_{y}^{\beta} L_{t}^{\gamma}\left(R u\left(\frac{x^{\alpha}}{\alpha}, \frac{y^{\beta}}{\beta}, \frac{t^{\gamma}}{\gamma}\right)\right) \\
& +L_{x}^{\alpha} L_{y}^{\beta} L_{t}^{\gamma}\left(N u\left(\frac{x^{\alpha}}{\alpha}, \frac{y^{\beta}}{\beta}, \frac{t^{\gamma}}{\gamma}\right)\right)=L_{x}^{\alpha} L_{y}^{\beta} L_{t}^{\gamma}\left(g\left(\frac{x^{\alpha}}{\alpha}, \frac{y^{\beta}}{\beta}, \frac{t^{\gamma}}{\gamma}\right)\right) .
\end{aligned}
$$

Step 2: divide by $s^{m}$, and apply the conformable inverse triple Laplace transform to equation (32); it reduces to

$$
u(x, y, t)=G(x, y, t)-L_{p}^{-1} L_{q}^{-1} L_{s}^{-1}\left\{s^{-m}\left[L_{x}^{\alpha} L_{y}^{\beta} L_{t}^{\gamma}\left(R u\left(\frac{x^{\alpha}}{\alpha}, \frac{y^{\beta}}{\beta}, \frac{t^{\gamma}}{\gamma}\right)\right)+L_{x}^{\alpha} L_{y}^{\beta} L_{t}^{\gamma}\left(N u\left(\frac{x^{\alpha}}{\alpha}, \frac{y^{\beta}}{\beta}, \frac{t^{\gamma}}{\gamma}\right)\right)\right]\right\}
$$

where $G(x, y, t)$ represents the term coming from the source term and prescribed initial conditions.

Step 3: considering the conformable triple Laplace transform decomposition method, let the solution of equation (29) be an infinite series

$$
u\left(\frac{x^{\alpha}}{\alpha}, \frac{y^{\beta}}{\beta}, \frac{t^{\gamma}}{\gamma}\right)=\sum_{n=0}^{\infty} u_{n}\left(\frac{x^{\alpha}}{\alpha}, \frac{y^{\beta}}{\beta}, \frac{t^{\gamma}}{\gamma}\right),
$$

and the nonlinear term can be decomposed as 


$$
N u\left(\frac{x^{\alpha}}{\alpha}, \frac{y^{\beta}}{\beta}, \frac{t^{\gamma}}{\gamma}\right)=\sum_{n=0}^{\infty} A_{n}
$$

where $A_{n}$ is called Adomian polynomials of $u_{1}, u_{2}, u_{3}, \ldots, u_{n}$, and it can be calculated by the following formula:

$$
A_{n}=\frac{1}{n !}\left[\frac{\partial^{n}}{\partial \sigma^{n}}\left(N \sum_{i=1}^{\infty} \sigma^{i} u_{i}\right)\right]_{\sigma=0}, \quad \text { where } n=0,1,2,3,4, \ldots
$$

Substituting equations (35) and (36) in equation (34), we have

$$
\sum_{n=0}^{\infty} u_{n}\left(\frac{x^{\alpha}}{\alpha}, \frac{y^{\beta}}{\beta}, \frac{t^{\gamma}}{\gamma}\right)=G(x, y, t)-L_{p}^{-1} L_{q}^{-1} L_{s}^{-1}\left\{s^{-m}\left[L_{x}^{\alpha} L_{y}^{\beta} L_{t}^{\gamma}\left(R \sum_{n=0}^{\infty} u_{n}\left(\frac{x^{\alpha}}{\alpha}, \frac{y^{\beta}}{\beta}, \frac{t^{\gamma}}{\gamma}\right)\right)+L_{x}^{\alpha} L_{y}^{\beta} L_{t}^{\gamma}\left(N \sum_{n=0}^{\infty} A_{n}\right)\right]\right\}
$$

Step 4: now, by comparing on both sides of equation

(37), we get

$$
\begin{aligned}
& u_{0}\left(\frac{x^{\alpha}}{\alpha}, \frac{y^{\beta}}{\beta}, \frac{t^{\gamma}}{\gamma}\right)=G(x, y, t), \\
& u_{1}\left(\frac{x^{\alpha}}{\alpha}, \frac{y^{\beta}}{\beta}, \frac{t^{\gamma}}{\gamma}\right)=-L_{p}^{-1} L_{q}^{-1} L_{s}^{-1}\left\{s^{-m}\left[L_{x}^{\alpha} L_{y}^{\beta} L_{t}^{\gamma}\left(R u_{0}\left(\frac{x^{\alpha}}{\alpha}, \frac{y^{\beta}}{\beta}, \frac{t^{\gamma}}{\gamma}\right)+N A_{0}\right)\right]\right\}, \\
& u_{2}\left(\frac{x^{\alpha}}{\alpha}, \frac{y^{\beta}}{\beta}, \frac{t^{\gamma}}{\gamma}\right)=-L_{p}^{-1} L_{q}^{-1} L_{s}^{-1}\left\{s^{-m}\left[L_{x}^{\alpha} L_{y}^{\beta} L_{t}^{\gamma}\left(R u_{1}\left(\frac{x^{\alpha}}{\alpha}, \frac{y^{\beta}}{\beta}, \frac{t^{\gamma}}{\gamma}\right)+N A_{1}\right)\right]\right\},
\end{aligned}
$$

and so on...

In general, we write the following recursive formula:

$$
u_{n+1}\left(\frac{x^{\alpha}}{\alpha}, \frac{y^{\beta}}{\beta}, \frac{t^{\gamma}}{\gamma}\right)=-L_{p}^{-1} L_{q}^{-1} L_{s}^{-1}\left\{s^{-m}\left[L_{x}^{\alpha} L_{y}^{\beta} L_{t}^{\gamma}\left(R u_{n}\left(\frac{x^{\alpha}}{\alpha}, \frac{y^{\beta}}{\beta}, \frac{t^{\gamma}}{\gamma}\right)+N A_{n}\right)\right]\right\}
$$

where $m=1,2,3$ and $n=0,1,2,3, \ldots$

At the end, we approximate the analytical solution as follows:

$$
u\left(\frac{x^{\alpha}}{\alpha}, \frac{y^{\beta}}{\beta}, \frac{t^{\gamma}}{\gamma}\right)=\sum_{n=0}^{\infty} u_{n}\left(\frac{x^{\alpha}}{\alpha}, \frac{y^{\beta}}{\beta}, \frac{t^{\gamma}}{\gamma}\right)
$$

\section{Applications}

In this section, a numerical experiment is done using the conformable triple Laplace decomposition method to solve nonlinear homogeneous and nonhomogeneous partial fractional differential equation in 3-dimensional space.
Example 1. To illustrate the proposed method, let us consider the following nonlinear partial fractional differential equation:

$$
\frac{\partial^{\gamma} u}{\partial t^{\gamma}}+6 u \frac{\partial^{\alpha+\beta} u}{\partial y^{\beta} \partial x^{\alpha}}+\frac{\partial^{2 \alpha} u}{\partial x^{2 \alpha}}=0
$$

with initial condition $u(x, y, 0)=x y$, and where $\alpha, \beta, \gamma \in(0,1], x, y, t \in[t 0, \infty)$.

Solution 1. Rewrite equation (41) as

$$
\frac{\partial^{\gamma} u}{\partial t^{\gamma}}=-6 u \frac{\partial^{\alpha+\beta} u}{\partial y^{\beta} \partial x^{\alpha}}-\frac{\partial^{2 \alpha} u}{\partial x^{2 \alpha}} .
$$


Taking the conformable triple Laplace transform on both sides of equation (42), we have

$$
L_{x}^{\alpha} L_{y}^{\beta} L_{t}^{\gamma}\left(\frac{\partial^{\gamma} u}{\partial t^{\gamma}}\right)=-L_{x}^{\alpha} L_{y}^{\beta} L_{t}^{\gamma}\left(6 u \frac{\partial^{\alpha+\beta} u}{\partial y^{\beta} \partial x^{\alpha}}+\frac{\partial^{2 \alpha} u}{\partial x^{2 \alpha}}\right)
$$

Recalling Theorem $5 \quad$ (c), $\quad L_{x}^{\alpha} L_{y}^{\beta} L_{t}^{\gamma}\left(\left(\partial^{\gamma} / \partial t^{\gamma}\right)\right.$ $(u(x, y, t)))=s U(p, q, s)-U(p, q, 0)$.

So, equation (43) reduces to

$$
s U(p, q, s)-U(p, q, 0)=-L_{x}^{\alpha} L_{y}^{\beta} L_{t}^{\gamma}\left(6 u \frac{\partial^{\alpha+\beta} u}{\partial y^{\beta} \partial x^{\alpha}}+\frac{\partial^{2 \alpha} u}{\partial x^{2 \alpha}}\right) .
$$

Since $u(x, y, 0)=x y$, we have

$$
U(p, q, 0)=\frac{1}{p^{2} q^{2}}
$$

Now, by applying the conformable inverse triple Laplace transform to equation (44) and using initial condition equation (45), we obtain from equation (44)

$$
u(x, y, t)=L_{p}^{-1} L_{q}^{-1} L_{s}^{-1}\left(\frac{1}{p^{2} q^{2} s}\right)-L_{p}^{-1} L_{q}^{-1} L_{s}^{-1}\left[\frac{1}{s} L_{x}^{\alpha} L_{y}^{\beta} L_{t}^{\gamma}\left(6 u \frac{\partial^{\alpha+\beta} u}{\partial y^{\beta} \partial x^{\alpha}}+\frac{\partial^{2 \alpha} u}{\partial x^{2 \alpha}}\right)\right]
$$

By applying the proposed method, in particular, equations (35)-(37), let $u_{0}(x, y, t)=x y$, and the recursive relation is given by

$$
u_{n+1}=-L_{p}^{-1} L_{q}^{-1} L_{s}^{-1}\left[\frac{1}{s} L_{x}^{\alpha} L_{y}^{\beta} L_{t}^{\gamma}\left(6 A_{n}+\frac{\partial^{2 \alpha} u_{n}}{\partial x^{2 \alpha}}\right)\right], \quad \text { where } n=0,1,2,3, \ldots,
$$

where $A_{n}$ is the Adomian polynomial to decompose the nonlinear terms by using the relation

$$
A_{n}=\frac{1}{n !}\left[\frac{\partial^{n}}{\partial \sigma^{n}} f\left(\sum_{i=0}^{\infty} \sigma^{i} u_{i}\right)\right]_{\sigma=0}, \quad \text { where } n=0,1,2,3,4, \ldots
$$

Let the nonlinear term be represented as

$$
f\left(u_{n}(x, y, t)\right)=u_{n} \frac{\partial^{\alpha+\beta} u_{n}}{\partial y^{\beta} \partial x^{\alpha}}
$$

Substituting equation (49) in equation (48), and also calculating $\left(\partial^{2 \alpha} u_{n} / \partial x^{2 \alpha}\right)$, the resulting expression of equation (47) reduces to the following.

For $n=0$,

$$
\begin{aligned}
A_{0} & =f\left(u_{0}\right)=\left(u_{0} \frac{\partial^{\alpha+\beta} u_{0}}{\partial y^{\beta} \partial x^{\alpha}}\right)_{\sigma=0}=x^{2-\alpha} y^{2-\beta}, \\
\frac{\partial^{2 \alpha} u_{0}}{\partial x^{2 \alpha}} & =\frac{\partial^{\alpha}}{\partial x^{\alpha}} \frac{\partial^{\alpha}}{\partial x^{\alpha}}(x y)=(1-\alpha) x^{1-2 \alpha} y .
\end{aligned}
$$

We have

$$
\begin{aligned}
& u_{1}=-L_{p}^{-1} L_{q}^{-1} L_{s}^{-1}\left[\frac{1}{s} L_{x}^{\alpha} L_{y}^{\beta} L_{t}^{\gamma}\left(6 x^{2-\alpha} y^{2-\beta}+(1-\alpha) x^{1-2 \alpha} y\right)\right], \\
& u_{1}=-6 x^{2-\alpha} y^{2-\beta} t-(1-\alpha) x^{1-2 \alpha} y t .
\end{aligned}
$$

For $n=1$,

$$
A_{1}=\frac{1}{1 !}\left[\frac{\partial}{\partial \sigma} f\left(u_{0}+\sigma u_{1}\right)\right]_{\sigma=0}=\frac{1}{1 !}\left[\frac{\partial}{\partial \sigma}\left\{\left(u_{0}+\sigma u_{1}\right)\left(\frac{\partial^{\alpha+\beta}}{\partial y^{\beta} \partial x^{\alpha}}\left(u_{0}+\sigma u_{1}\right)\right)\right\}\right]_{\sigma=0}
$$

Using equation (51), $u_{0}$, and $u_{1}$ and simplifying, equation (52) becomes 
International Journal of Differential Equations

11

$$
\begin{aligned}
A_{1}= & u_{1} \frac{\partial^{\alpha+\beta}\left(u_{0}\right)}{\partial y^{\beta} \partial x^{\alpha}}+u_{0} \frac{\partial^{\alpha+\beta}\left(u_{1}\right)}{\partial y^{\beta} \partial x^{\alpha}} \\
= & \left(-6 x^{2-\alpha} y^{2-\beta} t-(1-\alpha) x^{1-2 \alpha} y t\right) x^{1-\alpha} y^{1-\beta} \\
& +\left(x y\left[-6(2-\alpha)(2-\beta) x^{2-2 \alpha} y^{2-2 \beta} t-(1-\alpha)(1-2 \alpha) x^{1-3 \alpha} y^{1-\beta} t\right]\right) \\
\frac{\partial^{2 \alpha} u_{1}}{\partial x^{2 \alpha}=} & {\left[-6(2-\alpha)(2-2 \alpha) x^{2-3 \alpha} y^{2-\beta} t-(1-\alpha)(1-2 \alpha)(1-3 \alpha) x^{1-4 \alpha} y t\right] . }
\end{aligned}
$$

We have

$$
\begin{aligned}
u_{2}= & -L_{p}^{-1} L_{q}^{-1} L_{s}^{-1}\left[\frac { 1 } { s } L _ { x } ^ { \alpha } L _ { y } ^ { \beta } L _ { t } ^ { \gamma } \left\{6\left(-6 x^{2-\alpha} y^{2-\beta} t-(1-\alpha) x^{1-2 \alpha} y t\right) x^{1-\alpha} y^{1-\beta}\right.\right. \\
& +6 x y\left[-6(2-\alpha)(2-\beta) x^{2-2 \alpha} y^{2-2 \beta} t-(1-\alpha)(1-2 \alpha) x^{1-3 \alpha} y^{1-\beta} t\right] \\
& \left.\left.+\left\{-6(2-\alpha)(2-2 \alpha) x^{2-3 \alpha} y^{2-\beta} t-(1-\alpha)(1-2 \alpha)(1-3 \alpha) x^{1-4 \alpha} y t\right\}\right\}\right] .
\end{aligned}
$$

Simplifying equation (54), we have

$$
\begin{aligned}
u_{2}= & {\left[18 x^{3-2 \alpha} y^{3-2 \beta}+3(1-\alpha) x^{2-3 \alpha} y^{2-\beta}\right.} \\
& +18(2-\alpha)(2-\beta) x^{3-2 \alpha} y^{3-2 \beta}+3(1-\alpha)(1-2 \alpha) x^{2-3 \alpha} y^{2-\beta} \\
& \left.+3(2-\alpha)(2-2 \alpha) x^{2-3 \alpha} y^{2-\beta}+\frac{1}{2}\left((1-\alpha)(1-2 \alpha)(1-3 \alpha) x^{1-4 \alpha} y\right)\right] t^{2} \\
= & \left\{18[1+(2-\alpha)(2-\beta)] x^{3-2 \alpha} y^{3-2 \beta}+3(3-2 \alpha)(2-2 \alpha) x^{2-3 \alpha} y^{2-\beta}+\frac{1}{2}(1-\alpha)(1-2 \alpha)(1-3 \alpha) x^{1-4 \alpha} y\right\} t^{2}
\end{aligned}
$$

For $n=2$,

$$
\begin{aligned}
A_{2} & =\frac{1}{2 !}\left[\frac{\partial^{2}}{\partial \sigma^{2}} f\left(u_{0}+\sigma u_{1}+6^{2} u_{2}\right)\right]_{\sigma=0} \\
& =\frac{1}{2 !}\left[\frac{\partial^{2}}{\partial \sigma^{2}}\left\{\left(u_{0}+\sigma u_{1}+6^{2} u_{2}\right)\left(\frac{\partial^{\alpha+\beta}}{\partial y^{\beta} \partial x^{\alpha}}\left(u_{0}+\sigma u_{1}+6^{2} u_{2}\right)\right)\right\}\right]_{\sigma=0} \\
& =\frac{1}{2 !}\left[2 u_{0} \frac{\partial^{\alpha+\beta}\left(u_{2}\right)}{\partial y^{\beta} \partial x^{\alpha}}+2 u_{1} \frac{\partial^{\alpha+\beta}\left(u_{1}\right)}{\partial y^{\beta} \partial x^{\alpha}}+2 u_{2} \frac{\partial^{\alpha+\beta}\left(u_{0}\right)}{\partial y^{\beta} \partial x^{\alpha}}\right] .
\end{aligned}
$$


Substituting $u_{0}, u_{1}$, and $u_{2}$ in equation (56) and simplifying, we obtain

$$
\begin{aligned}
A_{2}= & {\left[(18[1+(2-\alpha)(2-\beta)]+18[1+(2-\alpha)(2-\beta)](3-2 \alpha)(3-2 \beta)+36(2-\alpha)(2-\beta)) x^{4-3 \alpha} y^{4-3 \beta} t^{2}\right.} \\
& +(3(2-2 \alpha)(3-2 \alpha)+3(2-2 \alpha)(3-2 \alpha)(2-3 \alpha)(2-\beta)+6(1-\alpha)(1-2 \alpha)+6(1-\alpha)(2-\alpha)(2-\beta)) x^{3-4 \alpha} y^{3-2 \beta} t^{2} \\
& \left.+\left(\frac{1}{2}(1-3 \alpha)+\frac{1}{2}(1-3 \alpha)(1-4 \alpha)+(1-\alpha)\right)(1-\alpha)(1-2 \alpha) x^{2-5 \alpha} y^{2-\beta} t^{2}\right] \\
\frac{\partial^{2 \alpha} u_{2}}{\partial x^{2 \alpha}=} & {\left[18[1+(2-\alpha)(2-\beta)](3-2 \alpha)(3-3 \alpha) x^{3-4 \alpha} y^{3-2 \beta} t^{2}\right.} \\
& +3(2-2 \alpha)(3-2 \alpha)(2-3 \alpha)(2-4 \alpha) x^{2-5 \alpha} y^{2-\beta} t^{2} \\
& \left.+\frac{1}{2}(1-\alpha)(1-2 \alpha)(1-3 \alpha)(1-4 \alpha)(1-5 \alpha) x^{1-6 \alpha} y t^{2}\right] .
\end{aligned}
$$

Therefore, we have

$$
\begin{aligned}
u_{3}= & -L_{p}^{-1} L_{q}^{-1} L_{s}^{-1}\left[\frac{1}{s} L_{x}^{\alpha} L_{y}^{\beta} L_{t}^{\gamma}\{6[(18[1+2(2-\alpha)(2-\beta)]+18[1+(2-\alpha)(2-\beta)](3-2 \alpha)(3-2 \beta)\right. \\
& +36(2-\alpha)(3-2 \alpha)) \\
& x^{4-3 \alpha} y^{4-3 \beta} t^{2}+6(3(2-2 \alpha)(3-2 \alpha)+3(2-2 \alpha)(3-2 \alpha)(2-3 \alpha)(2-\beta)+6(1-\alpha)(1-2 \alpha)+6(1-\alpha)(2-\alpha)(2-\beta)) \\
& x^{3-4 \alpha} y^{3-2 \beta} t^{2}+\left(\frac{1}{2}(1-3 \alpha)+\frac{1}{2}(1-3 \alpha)(1-4 \alpha)+(1-\alpha)\right)(1-\alpha)(1-2 \alpha) x^{2-5 \alpha} y^{2-\beta} t^{2} \\
& +\left[18[1+(2-\alpha)(2-\beta)](3-2 \alpha)(3-3 \alpha) x^{3-4 \alpha} y^{3-2 \beta} t^{2}\right. \\
& +3(2-2 \alpha)(3-2 \alpha)(2-3 \alpha)(2-4 \alpha) x^{2-5 \alpha} y^{2-\beta} t^{2} \\
& \left.\left.\left.+\frac{1}{2}(1-\alpha)(1-2 \alpha)(1-3 \alpha)(1-4 \alpha)(1-5 \alpha) x^{1-6 \alpha} y t^{2}\right]\right\}\right]
\end{aligned}
$$

Simplifying equation (58), we have

$$
\begin{aligned}
u_{3}= & -\left\{2 \left((18[1+(2-\alpha)(2-\beta)]+18[1+(2-\alpha)(2-\beta)](3-2 \alpha)(3-2 \beta)+36(2-\alpha)(2-\beta)) x^{4-3 \alpha} y^{4-3 \beta}\right.\right. \\
& +(3(2-2 \alpha)(3-2 \alpha)+3(2-2 \alpha)(3-2 \alpha)(2-3 \alpha)(2-\beta)+6(1-\alpha)(1-2 \alpha) \\
& +6(1-\alpha)(2-\alpha)(2-\beta)) x^{3-4 \alpha} y^{3-2 \beta} \\
& \left.+\left(\frac{1}{2}(1-3 \alpha)+\frac{1}{2}(1-3 \alpha)(1-4 \alpha)+(1-\alpha)\right)(1-\alpha)(1-2 \alpha) x^{2-5 \alpha} y^{2-\beta}\right) \\
& +\left(6[1+(2-\alpha)(2-\beta)](3-2 \alpha)(3-3 \alpha) x^{3-4 \alpha} y^{3-2 \beta}+(2-2 \alpha)(3-2 \alpha)(2-3 \alpha)(2-4 \alpha) x^{2-5 \alpha} y^{2-\beta}\right. \\
& \left.\left.+\frac{1}{6}(1-\alpha)(1-2 \alpha)(1-3 \alpha)(1-4 \alpha)(1-5 \alpha) x^{1-6 \alpha} y\right)\right\} t^{3},
\end{aligned}
$$


and so on....

The approximate series solution is expressed as

$$
\begin{aligned}
& u(x, y, t)=\left[x y-\left\{6 x^{2-\alpha} y^{2-\beta}+(1-\alpha) x^{1-2 \alpha} y\right\} t\right. \\
& +\left\{18[1+(2-\alpha)(2-\beta)] x^{3-2 \alpha} y^{3-2 \beta}+3(3-2 \alpha)(2-2 \alpha) x^{2-3 \alpha} y^{2-\beta}+\frac{1}{2}(1-\alpha)(1-2 \alpha)(1-3 \alpha) x^{1-4 \alpha} y\right\} t^{2} \\
& -\left\{2 \left((18[1+(2-\alpha)(2-\beta)])+18[1+(2-\alpha)(2-\beta)](2-\alpha)(2-\beta)(3-2 \alpha)(3-2 \beta) x^{4-3 \alpha} y^{4-3 \beta}\right.\right. \\
& +36(2-\alpha)(2-\beta)+(3(2-2 \alpha)(3-2 \alpha) \\
& +3(2-2 \alpha)(3-2 \alpha)(2-3 \alpha)(2-\beta)+6(1-\alpha)(1-2 \alpha)+6(1-\alpha)(2-\alpha)(2-\beta)) x^{3-4 \alpha} y^{3-2 \beta} \\
& \left.+\left(\frac{1}{2}(1-3 \alpha)+\frac{1}{2}(1-3 \alpha)(1-4 \alpha)+(1-\alpha)\right)(1-\alpha)(1-2 \alpha) x^{2-5 \alpha} y^{2-\beta}\right) \\
& +\left(6[1+(2-\alpha)(2-\beta)](3-2 \alpha)(3-3 \alpha) x^{3-4 \alpha} y^{3-2 \beta}+(2-2 \alpha)(3-2 \alpha)(2-3 \alpha)\right. \\
& \left.\left.\left.(2-4 \alpha) x^{2-5 \alpha} y^{2-\beta}+\frac{1}{6}(1-\alpha)(1-2 \alpha)(1-3 \alpha)(1-4 \alpha)(1-5 \alpha) x^{1-6 \alpha} y\right)\right\} t^{3}\right]
\end{aligned}
$$

From equation (60), if we consider $\alpha=\beta=\gamma=1$, then the solution of equation (41) reduces to

$$
u(x, y, t)=x y-6 x y t+36 x y t^{2}-216 x y t^{3}+\cdots=\frac{x y}{1+6 t} .
$$

Figures 1 and 2 show the 3D graphical representations of equation (60) with various values of $\alpha$ and $\beta$.

Example 2. Consider a nonlinear nonhomogeneous partial fractional differential equation, for $\alpha, \beta, \gamma \in$ $(0,1], x, y, t \in[t 0, \infty)$.

$$
\frac{\partial^{\beta} u}{\partial y^{\beta}} \frac{\partial^{\gamma} u}{\partial t^{\gamma}}-\frac{\partial^{2 \alpha} u}{\partial x^{2 \alpha}}=u(x, y, t)
$$

with initial conditions

$$
\begin{gathered}
u(0, y, t)=y t, \\
u_{x}(0, y, t)=-1 .
\end{gathered}
$$

Solution 2. Rewrite equation (62) as

$$
\frac{\partial^{2 \alpha} u}{\partial x^{2 \alpha}}=\frac{\partial^{\beta} u}{\partial y^{\beta}} \frac{\partial^{\gamma} u}{\partial t^{\gamma}}-u(x, y, t)
$$

Imposing the conformable triple Laplace transform to both sides,

$$
L_{x}^{\alpha} L_{y}^{\beta} L_{t}^{\gamma}\left(\frac{\partial^{2 \alpha} u}{\partial x^{2 \alpha}}\right)=L_{x}^{\alpha} L_{y}^{\beta} L_{t}^{\gamma}\left(\frac{\partial^{\beta} u}{\partial y^{\beta}} \frac{\partial^{\gamma} u}{\partial t^{\gamma}}-u(x, y, t)\right)
$$

Recalling Theorem 5 (d),

$$
L_{x}^{\alpha} L_{y}^{\beta} L_{t}^{\gamma}\left(\frac{\partial^{2 \alpha}}{\partial x^{2 \alpha}}(u(x, y, t))\right)=p^{2} U(p, q, s)-p U(0, q, s)-U_{x}(0, q, s) .
$$

Equation (66) becomes 


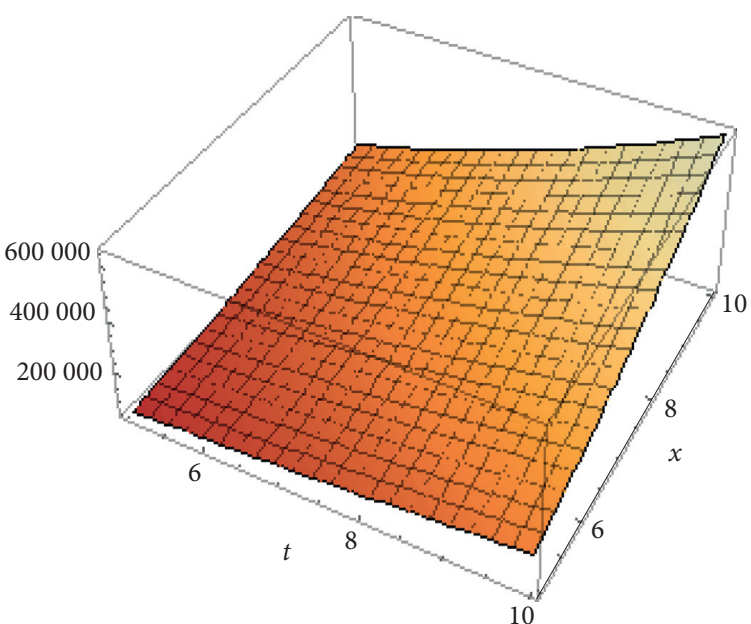

Figure 1: 3D plot of equation (60) for $\alpha=0.50 ; \beta=0.25 ; y=1$.

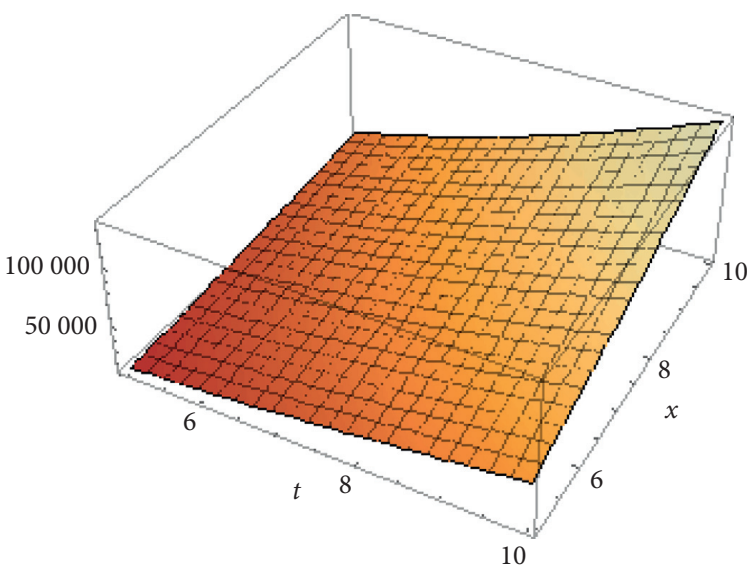

FIGURE 2: 3D plot of equation (60) for $\alpha=0.75 ; \beta=0.90 ; y=1$.

$$
p^{2} U(p, q, s)=p U(0, q, s)+U_{x}(0, q, s)+L_{x}^{\alpha} L_{y}^{\beta} L_{t}^{\gamma}\left(\frac{\partial^{\beta} u}{\partial y^{\beta}} \frac{\partial^{\gamma} u}{\partial t^{\gamma}}-u(x, y, t)\right) .
$$

Using initial condition (equation (63)) and taking the inverse triple Laplace transform on equation (67), we obtain

$$
\begin{aligned}
& u(x, y, t)=\left[L_{p}^{-1} L_{q}^{-1} L_{s}^{-1}\left(\frac{1}{p q^{2} s^{2}}-\frac{1}{p^{2} q s}\right)+L_{p}^{-1} L_{q}^{-1} L_{s}^{-1}\left(\frac{1}{p^{2}} L_{x}^{\alpha} L_{y}^{\beta} L_{t}^{\gamma}\left(\frac{\partial^{\beta} u}{\partial y^{\beta}} \frac{\partial^{\gamma} u}{\partial t^{\gamma}}-u(x, y, t)\right)\right)\right] \\
& u(x, y, t)=y t-x+L_{p}^{-1} L_{q}^{-1} L_{s}^{-1}\left(\frac{1}{p^{2}} L_{x}^{\alpha} L_{y}^{\beta} L_{t}^{\gamma}\left(\frac{\partial^{\beta} u}{\partial y^{\beta}} \frac{\partial^{\gamma} u}{\partial t^{\gamma}}-u(x, y, t)\right)\right) .
\end{aligned}
$$




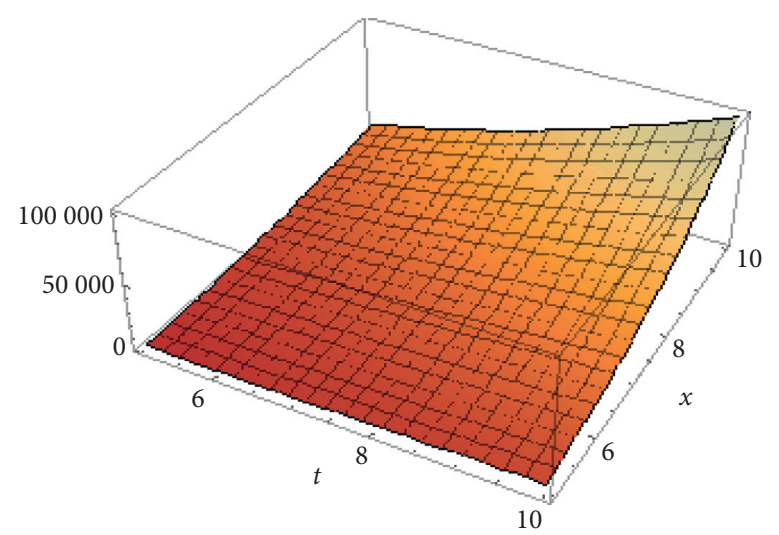

Figure 3: 3D plot of equation (73) for $\gamma=0.50 ; \beta=0.25 ; y=1$.

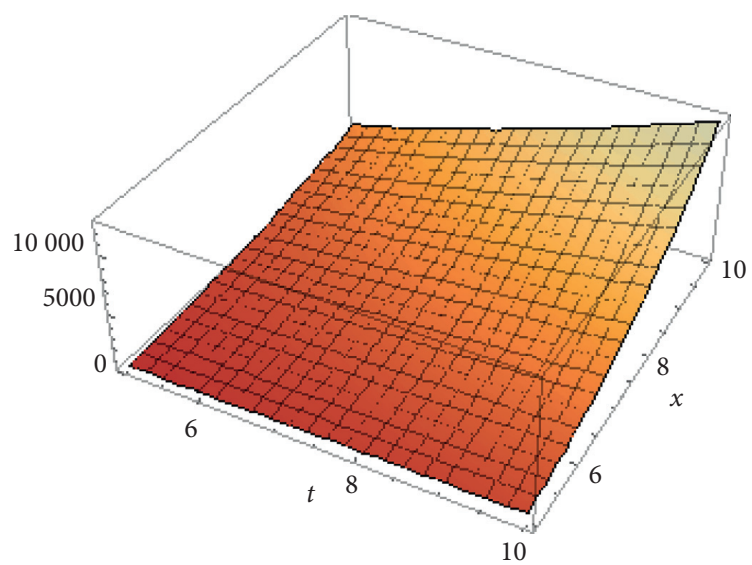

Figure 4: 3D plot of equation (73) for $\gamma=0.75 ; \beta=0.90 ; y=1$.

By applying the proposed method, we have the Let $u_{0}=y t-x$, and the recursive relation is following.

$$
\begin{aligned}
u_{n+1} & =L_{p}^{-1} L_{q}^{-1} L_{s}^{-1}\left(\frac{1}{p^{2}} L_{x}^{\alpha} L_{y}^{\beta} L_{t}^{\gamma}\left(\frac{\partial^{\beta} u_{n}}{\partial y^{\beta}} \frac{\partial^{\gamma} u_{n}}{\partial t^{\gamma}}-u_{n}\right)\right) \\
& =L_{p}^{-1} L_{q}^{-1} L_{s}^{-1}\left(\frac{1}{p^{2}} L_{x}^{\alpha} L_{y}^{\beta} L_{t}^{\gamma}\left(A_{n}-u_{n}\right)\right)
\end{aligned}
$$

where $A_{n}$ is the Adomian polynomial to decompose the nonlinear terms by using the following relation:

$$
A_{n}=\frac{1}{n !}\left[\frac{\partial^{n}}{\partial \sigma^{n}} f\left(\sum_{i=0}^{\infty} \sigma^{i} u_{i}\right)\right]_{\sigma=0}, \quad \text { where } n=0,1,2,3,4, \ldots
$$


Let the nonlinear term be represented as

$$
f(u)=\frac{\partial^{\beta} u}{\partial y^{\beta}} \frac{\partial^{\gamma} u}{\partial t^{\gamma}}
$$

Note that, in Adomian relation, the linear term can be considered, in place of nonlinear. So, you may take $f(u)=u$, and it leads to the same answer. So, this method is also valid for linear partial fractional differential equation.

For $n=0$,

$$
\begin{aligned}
& A_{0}=f\left(u_{0}\right) \\
& u_{1}=L_{p}^{-1} L_{q}^{-1} L_{s}^{-1}\left(\frac{1}{p^{2}} L_{x}^{\alpha} L_{y}^{\beta} L_{t}^{\gamma}\left(y^{2-\beta} t^{2-\gamma}-(y t-x)\right)\right) \\
& =\frac{x^{2}}{2} y^{2-\beta} t^{2-\gamma}-\frac{x^{2}}{2} y t+\frac{x^{3}}{3 !} \\
& n=1 \text {, } \\
& A_{1}=\frac{\partial}{\partial \sigma}\left[f\left(u_{0}+\sigma u_{1}\right)\right] \\
& =\frac{\partial}{\partial \sigma}\left(\frac{\partial^{\beta}\left(u_{0}+\sigma u_{1}\right)}{\partial y^{\beta}} \frac{\partial^{\gamma}\left(u_{0}+\sigma u_{1}\right)}{\partial t^{\gamma}}\right) \\
& =\frac{\partial^{\beta} u_{0}}{\partial y^{\beta}} \frac{\partial^{\gamma} u_{1}}{\partial t^{\gamma}}+\frac{\partial^{\beta} u_{1}}{\partial y^{\beta}} \frac{\partial^{\gamma} u_{0}}{\partial t^{\gamma}} \\
& A_{1}=\left[\left((2-\gamma) \frac{x^{2}}{2} y^{3-2 \beta} t^{3-2 \gamma}-\frac{x^{2}}{2} y^{2-\beta} t^{2-\gamma}\right)+\left((2-\beta) \frac{x^{2}}{2} y^{3-2 \beta} t^{3-2 \gamma}-\frac{x^{2}}{2} y^{2-\beta} t^{2-\gamma}\right)\right], \\
& u_{2}=L_{p}^{-1} L_{q}^{-1} L_{s}^{-1}\left(\frac { 1 } { p ^ { 2 } } L _ { x } ^ { \alpha } L _ { y } ^ { \beta } L _ { t } ^ { \gamma } \left(\left\{\left((2-\gamma) \frac{x^{2}}{2} y^{3-2 \beta} t^{3-2 \gamma}-\frac{x^{2}}{2} y^{2-\beta} t^{2-\gamma}\right)\right.\right.\right. \\
& \left.+\left((2-\beta) \frac{x^{2}}{2} y^{3-2 \beta} t^{3-2 \gamma}-\frac{x^{2}}{2} y^{2-\beta} t^{2-\gamma}\right)\right\} \\
& \left.\left.-\left(\frac{x^{2}}{2} y^{2-\beta} t^{2-\gamma}-\frac{x^{2}}{2} y t+\frac{x^{3}}{3 !}\right)\right)\right) \\
& =\left[\left((2-\gamma) \frac{x^{4}}{4 !} y^{3-2 \beta} t^{3-2 \gamma}-\frac{x^{4}}{4 !} y^{2-\beta} t^{2-\gamma}\right)+\left((2-\beta) \frac{x^{4}}{4 !} y^{3-2 \beta} t^{3-2 \gamma}-\frac{x^{4}}{4 !} y^{2-\beta} t^{2-\gamma}\right)-\left(\frac{x^{4}}{4 !} y^{2-\beta} t^{2-\gamma}-\frac{x^{4}}{4 !} y t+\frac{x^{5}}{5 !}\right)\right], \\
& u_{2}=(4-\gamma-\beta) \frac{x^{4}}{4 !} y^{3-2 \beta} t^{3-2 \gamma}-\frac{x^{4}}{8} y^{2-\beta} t^{2-\gamma}+\frac{x^{4}}{4 !} y t-\frac{x^{5}}{5 !},
\end{aligned}
$$

and so on...

The approximate series solution is written as

$$
u(x, y, t)=\left[(y t-x)+\left(\frac{x^{2}}{2} y^{2-\beta} t^{2-\gamma}-\frac{x^{2}}{2} y t+\frac{x^{3}}{3 !}\right)+\left((4-\gamma-\beta) \frac{x^{4}}{4 !} y^{3-2 \beta} t^{3-2 \gamma}-\frac{x^{4}}{8} y^{2-\beta} t^{2-\gamma}+\frac{x^{4}}{4 !} y t-\frac{x^{5}}{5 !}\right)+\cdots\right]
$$


For $\alpha, \beta, \gamma \in(0,1,[x, y, t \in] 0, \infty)$.

From equation (73), note that for $\alpha, \beta, \gamma=1$, the solution of equation (63) reduces to

$$
u(x, y, t)=y t-\left(x-\frac{x^{3}}{3 !}+\frac{x^{5}}{5 !}+\ldots\right)=y t-\sin x .
$$

Figures 3 and 4 show the 3D graphical representations of equation (74) with various values of $\gamma$ and $\beta$.

\section{Conclusion}

In this work, the conformable triple Laplace transform has been investigated using all our obtained novel results and theorems. The new conformable triple Laplace transform decomposition method is applied to find the solution of linear and nonlinear homogeneous and nonhomogeneous partial fractional differential equations. A numerical experiment has been conducted using this proposed method. This proposed method can be applied for simultaneous two or more than two linear and nonlinear partial fractional differential equations. Note that, if we take $\alpha, \beta, \gamma=1$, in Examples 1 and 2, we obtain an exact solution which was considered in [27]. Our results shed the light on the significance of exploring new generalized methods for solving partial differential equations, particularly nonlinear ones, due to the essential need to explore new analytical solutions to understand the dynamics of solutions for such important equations in physics and engineering.

\section{Data Availability}

No data were used to support this study.

\section{Conflicts of Interest}

The authors declare that there are no conflicts of interest regarding the publication of this study.

\section{References}

[1] A. A. Kilbas, O. I. Marichev, and S. G. Samko, Fractional Integrals and Derivatives (Theory and Applications), p. 1, Taylor \& Francis, Oxfordshire, UK, 1993.

[2] R. Khalil, M. Al Horani, A. Yousef, and M. Sababheh, "A new definition of fractional derivative," Journal of Computational and Applied Mathematics, vol. 264, pp. 65-70, 2014.

[3] T. Abdeljawad, "On conformable fractional calculus," Journal of Computational and Applied Mathematics, vol. 279, pp. 57-66, 2015.

[4] A. A. Abdelhakim and J. A. T. Machado, "A critical analysis of the conformable derivative," Nonlinear Dynamics, vol. 95, no. 4, pp. 3063-3073, 2019.

[5] A. Atangana, B. Dumitru, and A. Ahmed, "New properties of conformable derivative," Open Mathematics, vol. 13, no. 1, 2015.

[6] Gözütok, N. Yazıcı, and U. Gözütok, "Multivariable conformable fractional calculus," 2017, http://arxiv.org/abs/1701. 00616.

[7] M. ALHorani and R. Khalil, "Total fractional differentials with applications to exact fractional differential equations,"
International Journal of Computer Mathematics, vol. 95, no. 67, pp. 1444-1452, 2018.

[8] F. Martínez, I. Martínez-Vidal, and S. Paredes, "Conformable Euler's theorem on homogeneous functions," Computational and Mathematical Methods, vol. 1, no. 5, Article ID e1048, 2019.

[9] D. R. Anderson and D. J. Ulness, "Newly defined conformable derivatives," Advances in Dynamical Systems and Applications, vol. 10, no. 2, pp. 109-137, 2015.

[10] M. Al Horani, M. Abu Hammad, and R. Khalil, "Variation of parameters for local fractional nonhomogenous linear differential equations," The Journal of Mathematics and Computer Science, vol. 16, no. 2, pp. 147-153, 2016.

[11] M. A. Hammad and R. Khalil, "Abel's formula and wronskian for conformable fractional differential equations," International Journal of Differential Equations and Applications, vol. 13, no. 3, 2014.

[12] F. Silva, D. Moreira, and M. Moret, "Conformable Laplace transform of fractional differential equations," Axioms, vol. 7, no. 3 , p. $55,2018$.

[13] M. Al Masalmeh, "Series method to solve conformable fractional ric-cati differential equations," International Journal of Applied Mathematical Research, vol. 6, no. 1, pp. 30-33, 2017.

[14] K. K. Ali, R. I. Nuruddeen, and K. R. Raslan, "New hyperbolic structures for the conformable time-fractional variant bussinesq equations," Optical and Quantum Electronics, vol. 50, no. 2, pp. 1-10, 2018.

[15] F. Jarad and T. Abdeljawad, "A modifi ed Laplace transform for certain generalized fractional operators," Results in Nonlinear Analysis, vol. 1, no. 2, pp. 88-98, 2018.

[16] M. Omran and A. Kiliçman, "Fractional double Laplace transform and its properties," in AIP Conference Proceedings vol. 1795, no. 1, p. 020021, AIP Publishing LLC, 2017.

[17] R. I. Nuruddeen, F. D. Zaman, and Y. F. Zakariya, “Analysing the fractional heat diffusion equation solution in comparison with the new fractional derivative by decomposition method," Malaya Journal of Matematik, vol. 7, no. 2, pp. 213-222, 2019.

[18] M. Bouaouid, K. Hilal, and S. Melliani, "Nonlocal telegraph equation in frame of the conformable time-fractional derivative," Advances in Mathematical Physics, vol. 2019, Article ID 7528937, 7 pages, 2019.

[19] O. Özkan and K. Ali, "On conformable double Laplace transform," Optical and Quantum Electronics, vol. 50, no. 2, pp. 1-9, 2018.

[20] H. Gadain, "Application of double natural decomposition method for solving singular one dimensional Boussinesq equation," Filomat, vol. 32, no. 12, pp. 4389-4401, 2018.

[21] S. A. El-Wakil and E. M. Abulwafa, "Formulation and solution of space-time fractional Boussinesq equation," Nonlinear Dynamics, vol. 80, no. 1, pp. 167-175, 2015.

[22] S. Zhang and H.-Q. Zhang, "Fractional sub-equation method and its applications to nonlinear fractional PDEs," Physics Letters A, vol. 375, no. 7, pp. 1069-1073, 2011.

[23] H. E. Gadain, "Solving coupled pseudo-parabolic equation using a modified double Laplace decomposition method," Acta Mathematica Scientia, vol. 38, no. 1, pp. 333-346, 2018.

[24] M. Kaabar, "Novel methods for solving the conformable wave equation," Journal of New Theory, vol. 31, pp. 56-85, 2020.

[25] W. Gao, G. Yel, G. Yel, H. Mehmet Baskonus, and C. Cattani, "Complex solitons in the conformable $(2+1)$-dimensional Ablowitz-Kaup-Newell-Segur equation," AIMS Mathematics, vol. 5, no. 1, pp. 507-521, 2020. 
[26] A. Kumar, E. Ilhan, E. Ilhan, A. Ciancio, G. Yel, and H. M. Baskonus, "Extractions of some new travelling wave solutions to the conformable Date-Jimbo-Kashiwara-Miwa equation," Aims Mathematics, vol. 6, no. 5, pp. 4238-4264, 2021.

[27] A. Mousa and T. M. Elzaki, "Solution of nonlinear partial differential equations by triple Laplace decomposition method," 2019.

[28] M. Z. Mohamed, T. M. Elzaki, M. S. Algolam, M. Eltaib, E. Abd, and A. E. Hamza, "New modified variational iteration Laplace transform method compares Laplace adomian decomposition method for solution time-partial fractional differential equations," Journal of Applied Mathematics, vol. 2021, Article ID 6662645, 10 pages, 2021.

[29] K. K. Ali, M. S. Osman, H. M. Baskonus, N. S. Elazabb, and E. İlhan, "Analytical and numerical study of the HIV-1 infection of CD4+ T-cells conformable fractional mathematical model that causes acquired immunodeficiency syndrome with the effect of antiviral drug therapy," Mathematical Methods in the Applied Sciences, 2020.

[30] M. Eslami, "Exact traveling wave solutions to the fractional coupled nonlinear Schrodinger equations," Applied Mathematics and Computation, vol. 285, pp. 141-148, 2016.

[31] J. R. Loh, P. Chang, and K. Gaik Tay, "New method for solving fractional partial integro-differential equations by combination of Laplace transform and resolvent kernel method," Chinese Journal of Physics, vol. 67, pp. 666-680, 2020.

[32] F. Martínez, I. Martínez, I. Martínez, M. K. A. Kaabar, and S. Paredes, "New results on complex conformable integral," AIMS Mathematics, vol. 5, no. 6, pp. 7695-7710, 2020.

[33] F. Martínez, I. Martínez, M. K. A. Kaabar, and S. Paredes, "On conformable Laplace's equation," Mathematical Problems in Engineering, vol. 2021, Article ID 5514535, 10 pages, 2021.

[34] F. Martínez, I. Martínez, M. K. A. Kaabar, and S. Paredes, "Generalized conformable mean value theorems with applications to multivariable calculus," Journal of Mathematics, vol. 2021, Article ID 5528537, 7 pages, 2021.

[35] H. Bulut, T. A. Sulaiman, H. M. Baskonus, H. Rezazadeh, M. Eslami, and M. Mirzazadeh, "Optical solitons and other solutions to the conformable space-time fractional FokasLenells equation," Optik, vol. 172, pp. 20-27, 2018.

[36] M. Yavuz and N. Özdemir, "New numerical techniques for solving fractional partial differential equations in conformable sense," in Non-integer Order Calculus and its Applications, pp. 49-62, Springer, Cham, Switzerland, 2017.

[37] M. Yavuz, "Novel solution methods for initial boundary value problems of fractional order with conformable differentiation," An International Journal of Optimization and Control: Theories \& Applications, vol. 8, no. 1, pp. 1-7, 2018.

[38] F. Evirgen, "Conformable fractional gradient based dynamic system for constrained optimization problem," Acta Physica Polonica Series A, vol. 132, no. 3-II, pp. 1066-1069, 2017.

[39] D. Avcı, B. B. İ. Eroğlu, and N. Özdemir, "Conformable heat equation on a radial symmetric plate," Thermal Science, vol. 1, no. 2, 2017.

[40] E. B. I. Eroglu, D. Avc1, and N. Özdemir, "Optimal control problem for a conformable fractional heat conduction equation," Acta Physica Polonica Series a, vol. 132, no. 3, pp. 658-662, 2017. 